\title{
On The Status Of Solar Wind During The Present - Era Of Weak Solar Cycles
}

\author{
Shahinaz Yousef and Amhed Hady \\ Astronomy and Meteorology Department, Faculty Of Science, Cairo University, Egypt \\ email: Shahinazyousef@yahoo.com \& aahady@cu.edu.eg
}

\begin{abstract}
It is suggested that we are already in the weak solar cycles series since the start of cycle 23 . The interplanetary magnetic filed and the solar wind speed and density are expected to drop considerably during the approaching second weak cycle number 24 and the following ones leading to inflation of the magnetosphere. The corona is also expected to cool down relative to normal cycles corona. A daily background coronal index is proposed. The mechanism of production of weak cycles is tied to the rapid rotation of the photospheric layer which is deeply rooted in the bottom of the convection zone. This rapid surface and subsurface rotation implies slower rotation of the tachocline. Slower dynamo rotation leads to reduction of the strength of the magnetic cycle. One of the very important sequences of the weak cycles, is the expected cooling of the Earths air and sea surface temperatures which would have negative effects on agriculture with increased drought-flood hazards. The reduction of solar UV flux can lead to the closure of the ozone hole on the long run.
\end{abstract}

Keywords. Solar wind, solar cycle, interplanetary magnetic field, coronal index, solar UV flux

\section{Introduction}

An important task which really has strong implications on us, the residents of the earth, is to study and predict the status of the solar wind in the coming few cycles. This status is surely linked to the general status of the coming solar cycles activity. Therefore, this invited paper will address the prediction of the coming solar cycles, looking backward at the status of solar wind during similar solar cycles and then looking forward for prediction purposes This paper will also address some vital terrestrial implications of being in this particular very special era of weak magnetic solar cycles.

\section{Variability Of The Coronal Temperature}

"And We made the temperature far away from the lamp (the sun) variable"13 Al Nabaa The holy Quran. Indeed, what is important is the amazing high temperature of the solar corona some $2000 \mathrm{~km}$ above the solar surface and beyond and its variability. It is this variability which controls the amount of solar X-ray, UV and EUV radiations that affect our terrestrial atmosphere. The UV radiation doubles between solar minimum and maximum while the X-ray flux vary by 100 folds (Lang 2001). It is thus very important to find a proper daily temperature index for the general corona. One way of doing so is to use the ratio of the daily background X-ray flux in the $44-60 \mathrm{~A}, 8-20 \mathrm{~A}$ and even shorter wavelength bands. In case of routine measurements of X-ray lines intensities, then their ratios and their $\mathrm{G}$ functions can be used for estimating the temperatures. It is suggested that several temperature indices can be estimated on routine basis 1- A Daily general Corona temperature Index Tg can be derived from the background X-ray flux at $44-60$ A to 8 - 20 A. 2- A Daily Active Region Temperature Index Tac can be derived from $1-$ $8 \mathrm{~A}$ to $8-20 \mathrm{~A}$. 3- A Flare temperature index can be derived from $0.5-3 \mathrm{~A}$ to $1-8 \mathrm{~A}$ 
Tfl for each X-ray flare 4- Coronal Hole Temperature Index Tch Or alternatively those temperature indices can be derived from the ratio of carefully chosen spectral lines. The advantage of using X-ray bands is that backward temperature indices can be estimated for decades. Continuations of GOES X-ray bands routine observations are vital. The present absence of the $44-60$ A from Soho data is a pity. Perhaps an alternative method can be derived for the missing period. Soho people are encouraged to find some way of estimating coronal temperature from their outstanding data archives. The ratio of the coronal red and green lines may also be used for earlier studies. A working group on solar coronal temperature indices is suggested as one of the tasks of the IAU commissions on solar physics and solar activity. Perhaps also a workshop with this title can be held with the presence of Soho and GOES scientists. An experiment with the task of measuring different coronal temperature indices is suggested in future solar space missions.

\section{The Wolf-Gleissberg Cycles, (WGCs)}

The Sun has three types of solar magnetic cycles (Nesme-Ribes et al. 1994); the Maunder minimum type, the normal 11 yr. cycles and the weak magnetic cycles mostly of 12-yr. duration. Those weak cycles occurred around 1800 (cycles 5 to 7), 1900 (cycles 12 to 14 and possibly 15) and 2000 ( cycles 23 to 25 and possibly 26), see Yousef $(1995,1998$, and 2003). Different types of solar cycles are shown in Fig 1. On smoothing, larger scale $(80-120$ yr.) solar magnetic cycles known as the Wolf-Gleissberg cycles (WGCs) become distinguished (Fig 2). A series of 3-4 weak cycles occur intermediate between two successive WGCs. Eventually the last WGC ended in 1996 - 1997 with the start of cycle 23, the first of the present weak cycles series. In addition, those WGCs generally have two maximums of strong normal cycles separated by one or two weak cycles, e.g. the strong solar cycles 19 and 21 were separated by the weak cycle number 20 . The solar spin rate was faster during the start of weak cycle series as evident from Fig. 3a for the weak cycles earlier than 1900 and for cycle 20 in the 1960s (Hoyt and Schatten1997after their reference). This faster rotation rate leads to a reduction in solar energy budget emitted from the sun in all wavelengths as well as solar wind flux, density, velocity and interplanetary magnetic field. Solar induced climate changes do occur at the turning points of those WGCs whenever there is a change in the solar spin rate. Solar induced climate changes do occur at the start, end of those weak cycles and following the WGC maximums (Yousef 2000a). In addition, the solar induced climate change can occur within the weak cycles series following the end of 22-year Hale magnetic cycle as shown in figs (2) and (3a), a concept that will be discussed later. The present solar cycle number 23 is the first of weak cycle series, the last solar induced climate change did occur around 1997 with the start of those weak cycles at the end turning point of WGC (Yousef 1995 and 2003).

\section{Tachocline And Variability Of Solar Spin Rate}

The solar differential rotation persists to the bottom of the connective zone. The rotation velocity becomes uniform from pole to pole nearly $1 / 3$ of the way to the core Lower down the rotation remains independent of latitude acting as if the sun were a solid body. Shearing motion along this interface may be the dynamo source of magnetism. The top of the radiative zone which rotates at one speed meet the overlying convective zone which spins faster in its equatorial middle. The roughly 20 million meter wide layer where these very different zones meet and shear against one another, called the tachocline, is the likely site of the solar dynamo, the source of sun's magnetism. The hot circulating gases, 
which are good conductors of electricity, generate electrical currents that create magnetic fields, these fields in turn sustain the generation of electricity. The dynamo amplifies and regenerates the sun's magnetic field. The strong fields eventually rise through the connective zone and emerge at the photosphere (Lang 2001and references therein) There is a temporal variation in the rotation of the sun in the base of the connective zone and close to the probable site of the solar dynamo in equatorial regions, the rotation speeds up, slows down and speeds up again with a period of 1.3 year. There are observed differences of the rotation rate from the mean at $0.72 \mathrm{R}$ and $0.63 \mathrm{R}$. When the lower gas speeds up, the upper gas slows down and vise versa. The anticipated 11-year signal is not observed. However, the amplitude of those variations seems to decrease at the solar maximum. It is intriguing that similar 1.3-year are observed in the surface sunspot data ( Kosovichev 2003 after his references. If we tie up those variations of rotation speed near the tachocline with those observed at the photosphere, it is thus evident that faster rotations of weak solar cycles means slower rotation at the tachocline, i.e. of the solar dynamo and this implies the decline of solar magnetism. And vice versa, for stronger more active solar cycles the equatorial photosphere is observed to rotate slower, implying that the solar dynamo spins faster and that the hot circulating ionized gases generate stronger electrical currents and thus creating stronger magnetic fields.

\section{1.3 year periodicity}

The long living coronal structures are related to complexes of solar activity and displays quasi periodic behavior( impulses of coronal activity) with periods of $1.0-1.5$ year in the axisymmetrical distribution of EUV and X-ray fluxes during the current cycle( Bumba 2003 and references therein). Bumba (2003) also reports after his references that on studying the wavelet spectra of the sunspot number, solar wind plasma, interplanetary magnetic field and Ap index for the years 1964 - 2000, the 1.3 years periodicity is found. The wavelet power of the 1.3-year period in velocity was maximum during the time interval of 1992 - 94. Similarly, in the case of the Ap-index data, the wavelet power of the 1.3-year period peaked around the years 1945 and 1992.

\section{Prediction of the State of solar Activity During The Next Few Decades}

Weak solar cycles occur at the bottom of Wolf-Gleissberg cycles tend to occur in series of 3-4 cycles. A single weak cycle also occur in between the two maximums of WolfGleissberg cycle. As we are already in the bottom of the WGc, with cycle 23 the first of weak cycles series (Yousef 1995, 98, 2003), the next two to three cycles are also expected to be weak. Owing to the 200-years cylicity of the sun, it is more likely that the status of the solar activity would be something like those weak cycles around 1800 . but as a matter of fact cycle 23 was more like cycle 20 and stronger than cycles 5 and 12 . Cycles 5 to 7 were more symmetric around maximum. Svalgaard (2005) also predicted that cycle 24 would be the lowest so far in the past 100 years with the maximum sunspot number around 75 .

It is worth mentioning that in the past era of weak solar activity around 1900, the level of Lake Victoria and other Equatorial lakes rose and fall in coherence sympathy with the second weak cycle and the following ones (Yousef 2003). 


\section{Characteristics Of The State Of Solar Activity During Weak Cycles}

A mildly to weak active sun is what we should expect in the coming decades. The following characteristics are most likely to prevail.

1. Drop of solar irradiance.

2. Drop of coronal temperature and coronal magnetic loops.

3. Drop in the flux of solar gamma rays, X-rays, UV, IR and radio waves.

4. Drop in the number and importance of solar flares.

5. Drop in the number of coronal mass ejections but increased percentage of Halo CME due to lowering of latitude. This may lead to more hurricanes.

6. Drop in solar wind flux and velocities leading to expansion of magnetosphere.

7. Drop in the strength of interplanetary magnetic field.

8. Increased invasion of cosmic rays to the earth thus increased cloud cover (Kirkby and Laaksonen 2000) We are going to discuss few of these implications in the context of solar wind.

\section{Prediction of the status of Solar wind and IMF During the next decades of Weak solar cycles}

Svalgaard et al. (2003) have succeeded in working out the near-earth interplanetary magnetic field since 1600 as shown in fig (3). They have also reconstructed solar wind speed since 1880 (fig4c). The number of 3-hour intervals per year where the aa-index was at its lowest possible values as shown in fig (4b). We study the IMF during the previous weak solar cycles series (cycles $5-7)$ and $(12-14)$ around 1800 and 1900 . In response to the drop of the level activity, the magnitude of IMF and the solar wind speed dropped. The IMF for cycles 5 and 6 is in the range of $4.5-6.5 \mathrm{nT}$ compared to $8.5 \mathrm{nT}$ for cycle 19 which is the maximum of the Wolf-Gleissberg cycle. Fig. (4) shows that the years $1900-$ 1902 seem to be in a class of their own with very low solar wind speed $(275-300 \mathrm{~km} / \mathrm{s})$. In order to understand what happened in 1900 - 1902 let us put the pieces together, compare figs (4a,b.c and d) starting from the sun down to earth

a) at those years solar equatorial rotation rate abruptly slowed down(fig. 4a).

b) the solar wind speed dropped down(fig. 4c)

c) Those years were also exceptionally quiet with more than half the time there was no measurable geomagnetic activity at all. (fig. 4b).

d) IMF dropped (fig. 3).

e) There was a sudden rise in the temperature of the small town Soria in Spain fig. 4d (Donaire 2000).

In the year 1900, slowing down of the equatorial rotation means that the Tachocline rotation speeds up. It is at those years that a solar induced climate change happened ending the previous one at 1878 . This 1878 earlier solar induced climate change occurred with the start of the weak cycle number 12 leading to about 5 degrees sudden temperature drop of little town Soria in Spain. Several examples of temperature drops in Edinburgh, Wakefield, and Greenwich and southern Mediterranean Stations are found in (Aesawy and Hasanean (1998) and Yousef (2000a). At those years also the IMF dropped down and it was a geomagnetic quiet period. But the sun was spinning much faster for a period of 22 years Hale magnetic cycle. Less irradiance reached the earth in all wave lengths of spectrum and this lead to a global cooling. So what counts here is the spin of the sun. 


\section{Implications of Weak cycles on The Earth}

During weak cycles, the coronal temperature drops leading to reduction of the $\mathrm{X}$ and UV flux. This has positive implication on the ozone hole. Less UV will lead to revival of the Algae in the Antarctic Ocean which will thus absorb more carbon dioxide from the atmosphere and dip it in the ocean (Yousef 1998). This will lead to cooling of the troposphere and warming of the stratosphere thus reducing the size of the stratospheric clouds and closure of the ozone hole during the era of weak cycles( Yousef and Al-Kuhaimi 1999). The reduction of solar irradiance is expected to lead to global cooling(Lean 1994). Air cooling will lead to reduction in agricultural productivity. Drop of Sea surface temperature as happened around 1900 in previous weak cycles series is expected to lead to increases La Nina events and thus more flood-drought hazards (Yousef 2000b and 2006).

\section{Conclusion}

How the sun really changes its status of magnetic activity, lies in the variability of its spin rate. On the photospheric level, such variability is observed (Hoyt and Schatten1997). Weak magnetic cycles spin faster than normal and very active cycles. Going deeper, the sun rotates as a rigid body while its convection zone shows differential rotation. It is at this interface, the Tachocline, where shearing occurs, the solar dynamo lies. Very important is the MDI and GONG finding that there is an inverse variation of the rotation rate in the tachocline $(0.72 \mathrm{R})$ and the upper radiative zone( $0.63 \mathrm{R})$ (Kosovichev 2003 and references therein). It seems that there is an inverse correlation between the photosphere and the convection zone rotation rates on one hand and the tachocline rotation on the other. One of the longer solar cycles which involves the periodicities of the weak and strong magnetic cycles is the $80-120$ Wolf-Gleissberg cycle. These very cycles indicate that the variation of rotation rate of the tachocline follows a cycle of $80-120$ years. Currently, the rotation rate of the tachocline is declining towards a lowest level. Cycle 23 is the first of a weak cycles series Yousef (2003). Cycle 23 is weak, however it is stronger than cycles 12 (the first of the previous weak cycles series) and much stronger than cycles (5 to 7 ) the earlier weak cycles series around 1800. Having defined the coming era as an era of weak solar cycles which started in $1996-97$. We can move to the next step of studying the solar wind at the two previous eras of weak solar cycles around 1800 and 1900. Indeed there was a decline of solar wind velocities at the time to about $300 \mathrm{~km} / \mathrm{s}$ and below and the IMF to $51.5 \mathrm{nT}$. We are already about $1 / 4$ of the way in the present era of weak cycles series. The consequences of the decline of the solar wind will lead to the expansion of the magnetosphere.

\section{Acknowledgements}

Our deep thanks and gratitude are due to our parents for support and devotion.

\section{References}

Aesawy, A.M. \& Hasanean, H.M. 1998, Theor. Appl. Climatol. 61, 55

Bumba, V. Proceedings of ISCS 2003. Solar Variability As An Input To The Earth's Environment.23-28 June 2003. Tatranska Lomnica, Slovak Republic. European Space Agency. ESA. Edited by A. Wilson, 3

Donaire, Jaun Jos Sanz 2000, Bulletin of the Egyptian Geographical Society, 73, 127

Hoyt, D.V. \& Schatten, K. 1997: The Role of The Sun In Climate Change. Oxford University Press 
Kirkby, J \& Laaksonen A. 2000: Solar Variability and Clouds. Solar Variability and Climate; Edited by Friis-Christensen, E., Fröhlich, C., Haigh, J.D., Schüssler M. \& Von Steiger R.. Space Sci.Rev 94, No1-2, 397

Kosovichev, A.G., 2003, Proceedings of ISCS 2003. Solar Variability As An Input To The Earth's Environment. 23 - 28 June 2003. Tatranska Lomnica, Slovak Republic. European Space Agency. ESA. Edited by A. Wilson

Lang, K.R. The Cambridge Encyclopedia Of The Sun. Cambridge University Press

Lean J. 1994: Solar Forcing On Global change. NATO ASI series 25. The solar engine and its influence on terrestrial atmosphere and climate, Edited by Nesme-Ribes, 163

Nesme-Ribes E., Sokoloff D., Ribes, J.C. \& Kremliovsky M. 1994: The Maunder minimum and the solar dynamo. NATO ASI series 25. The solar engine and its influence on tereestrial atmosphere and climate, Edited by Nesme-Ribes, 527

Svalgaard L., Cliver,E.W. \& LeSager,P.2003:Determination of Interplanetary Magnetic Field Strength, Solar Wind Speed and EUV Irradiance, 1890-Present. Proceedings of ISCS, European Space Agency ESA. SP-535. Solar Variability as an input to The Earth's Environment, 23 - 28 June, Tatranska Lomnica, Slovak Republic, pp $15-24$

Svalgaard L., Cliver,E.W. \& Kamide,Y. 2005: Sunspot cycle 24; smallest cycle in 100 years. Geophysical Res Lett. 32, L01104

Yousef, Shahinaz M. 1995: The Downturn of Solar Activity During The Forthcoming Three Cycles. Bull: Fac. Sc. Cairo Univ., 63, 185

Yousef Shahinaz M. 1998: A Warning Of Solar Inactivity During the Next Few Decades and Its Influence On IMF And Cosmic Rays. Proceedings of the Third SOLTIP Symposium, Oct 1996. Beijing, 569

Yousef Shahinaz M. 2000a: "The Solar Wolf-Gleissberg Cycle And Its Influence On The Earth" in the Proceedings of the International Conference on the Environmental Hazards Mitigation, Cairo Sept. 2000. Conferences of Virtual Academia

Yousef Shahinaz M. 2000b: An Overview Of Flood-Drought Hazards In The 21st Century. WMO International Workshop On Long Range Forecasting and Its Applications., P81. Cairo, January $23-27$

Yousef, Shahinaz M. 2003: Cycle 23, The First of Weak Solar Cycles Series and the Serious Implications on Some Sun-Earth Connections. Proceedings of ISCS, European Space Agency ESA. SP-535. Solar Variability as an input to The Earth's Environment, 23 - 28 June, Tatranska Lomnica, Slovak Republic, 177

Yousef, Shahinaz M. 2006: 80 - 120 yr. Long Term Solar Induced Effects on the Earth, Past and Predictions.Physics and Chemistry of the Earth

Yousef, Shahinaz M. and Al-Kuhaimi 1999: Expected drop in solar ultraviolet flux during the coming three decades and ozone hole recovery. Journal of Enviromental Siences 18. 149 